\title{
Linking the Meaning of Programs to What the Compiler Can Verify
}

\author{
Egon Börger \\ Università di Pisa, Dipartimento di Informatica, I-56125 Pisa, Italy \\ boerger@di.unipi.it
}

\begin{abstract}
We formulate some research and development challenges that relate what a verifying compiler can verify to the definition and analysis of the application-content of programs, where the analysis comprises both experimental validation and mathematical verification. We also point to a practical framework to deal with theses challenges, namely the Abstract State Machines (ASM) method for high-level system design and analysis. We explain how it allows one to bridge the gap between informal requirements and detailed code by combining application-centric experimentally validatable system modeling with mathematically verifiable refinements of abstract models to compiler-verifiable code.
\end{abstract}

This paper is a position paper, triggered by the formulation of the program verifier challenge in 46]. By its definition, Hoare's challenge is focussed on the correctness of programs: software representations of computer-based systems, tobe-compiled by a verifying compiler. As a consequence, "the criterion of correctness is specified by types, assertions and other redundant annotations associated with the code of the program", where "the compiler will work in combination with other program development and testing tools, to achieve any desired degree of confidence in the structural soundness of the system and the total correctness of its more critical components." [46] Compilable code however is the result of two program development activities, which have to be checked too:

- turning the requirements into ground models, accurate "blueprints" of the to-be-implemented piece of "real world", which define the application-centric meaning of programs in an abstract and precise form, prior to coding,

- linking ground models to compilable code by a series of refinements, which introduce step by step the details resulting from the design decisions for the implementation.

We propose to broaden the program verifier challenge by relating the verification of the correctness for compilable programs to the experimental validation of the application-domain-based semantical correctness for ground models and to the mathematical verification of their refinements to compilable code, using Abstract State Machine (ASM) ground models [1] (Sect. 1) and ASM refinements [12] (Sect.2). This leads us to formulate a broadening of Hoare's challenge, together with a series of milestones towards the overall goal (Sect. 4). 


\section{ASM Ground Models (System Blueprints): A Semantical Foundation for Program Verification}

Compilable programs, though often considered as the true definition of the system they represent, in many complex applications do however not "ground the design in reality", since they provide no correspondence between the extra-logical theoretical terms appearing in the code and their empirical interpretation, as requested by a basic principle of Carnap's analysis of scientific theories [24]. By ground models for software systems I mean mathematical application-centric models, which define what Brooks 23 calls "the conceptual construct" or the "essence" of code for a computer-based system and thus "ground the design in reality". Ground models are the result of the notoriously difficult and error prone elicitation of requirements (see [45 47]), largely a formalization and clarification task realizing the transition from mostly natural-language problem descriptions to a sufficiently precise, unambiguous, consistent, complete and minimal formulation, which represents the algorithmic content of the software contract.

By its epistemological role of relating some piece of "reality" to a linguistic description, the fundamental concept of ground model has no purely mathematical definition, though it can be given a scientific definition in terms of basic epistemological concepts which have been elaborated for empirical sciences by analytic philosophers, see for example [4344. We limit ourselves here to cite from [1] the essential properties which characterize the notion of ground models and can all be satisfied by ASM ground models. Ground models must be:

- precise at the appropriate level of detailing yet flexible, to satisfy the required accuracy exactly, without adding unnecessary precision;

- simple and concise to be understandable and acceptable as contract by both domain experts and system designers. ASM ground models allow one to achieve this property mainly by avoiding any extraneous encoding and by reflecting "directly", through the abstractions, the structure of the real-world problem. This makes ground models manageable for inspection and analysis, helps designers to resolve the "lack of scientific understanding on the part of their customers (and themselves)" [46, p.66] and enables experts to "clearly explain why ... systems indeed work correctly" 3];

- abstract (minimal) yet complete. Completeness means that every semantically relevant feature is present, that all contract benefits and obligations are mentioned and that there are no hidden clauses. In particular, a ground model must contain as interface all semantically relevant parameters concerning the interaction with the environment, and where appropriate also the basic architectural system structure. The completeness property "forces" the requirements engineer, as much as this is possible, to produce a model which is "closed" modulo some "holes", which are however explicitly delineated, including a statement of the assumptions made for them at the abstract level and to be realized through the detailed specification left for later refinements. Model closure implies that no gap in the understanding of "what to build" is left, that every relevant portion of implicit domain knowledge has been 
made explicit and that there is no missing requirement-avoiding a typical type of software errors that are hard to detect at the level of compilable code [53, Fact 25]. Minimality means that the model abstracts from details that are relevant either only for the further design or only for a portion of the application domain which does not influence the system to be built;

- validatable (see [45) and thus in principle falsifiable by experiment and rigorous analysis, satisfying the basic Popperian criterion for scientific models [52;

- equipped with a simple yet precise semantical foundation as a prerequisite for rigorous analysis and reliable tool support.

\section{ASM Refinements: Management of Design Decisions (Documentation and Verification)}

The ASM refinement notion I have proposed 1 generalizes Wirth's and Dijkstra's classical refinement method 6927. Using stepwise ASM refinements offers the practitioner a technique to cope with the "explosion of 'derived requirements' (the requirements for a particular design solution) caused by the complexity of the solution process" and encountered "when moving from requirements to design" [53. Fact 26], a process that precedes the definition of compilable code. The ASM refinement method supports practical system validation and verification techniques that split checking complex detailed properties into a series of simpler checks of more abstract properties and their correct refinement, following the path the designer has chosen to rigorously link through various levels of abstraction the system architect's view (at the abstraction level of a blueprint) to the programmer's view (at the level of detail of compilable code). Successive ASM refinements also provide a systematic code development documentation, including behavioral information by state-based abstractions and leading to "further improvements to quality and functionality of the code ... by good documentation of the internal interfaces" [46, p.66].

In choosing how to refine an ASM $M$ to an ASM $M^{*}$, one has the freedom to define the following items, as illustrated by Fig. 1:

- a notion (signature and intended meaning) of refined state,

- a notion of states of interest and of correspondence between $M$-states $S$ and $M^{*}$-states $S^{*}$ of interest, i.e. the pairs of states in the runs one wants to relate through the refinement, including usually the correspondence of initial and (if there are any) of final states,

- a notion of abstract computation segments $\tau_{1}, \ldots, \tau_{m}$, where each $\tau_{i}$ represents a single $M$-step, and of corresponding refined computation segments $\sigma_{1}, \ldots, \sigma_{n}$, of single $M^{*}$-steps $\sigma_{j}$, which in given runs lead from corresponding states of interest to (usually the next) corresponding states of interest (the resulting diagrams are called $(m, n)$-diagrams and the refinements $(m, n)$-refinements),

${ }^{1}$ The proposal goes back to 6/7/9 where it was used to define what became the ISO standard of Prolog [15]. For a recent survey see [12]. 


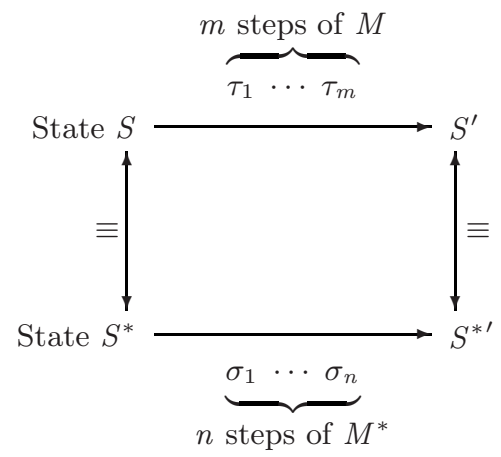

With an equivalence notion $\equiv$ between data in locations of interest in corresponding states.

Fig. 1. The ASM refinement scheme

- a notion of locations of interest and of corresponding locations, i.e. pairs of (possibly sets of) locations one wants to relate in corresponding states,

- a notion of equivalence $\equiv$ of the data in the locations of interest; these local data equivalences usually accumulate to a notion of equivalence of corresponding states of interest.

Once the notions of corresponding states and of their equivalence have been determined, one can define that $M^{*}$ is a correct refinement of $\mathrm{M}$ if and only if every (infinite) refined run simulates an (infinite) abstract run with equivalent corresponding states. More precisely: fix any notions $\equiv$ of equivalence of states and of initial and final states. An ASM $M^{*}$ is called a correct refinement of an ASM $M$ if and only if for each $M^{*}$-run $S_{0}^{*}, S_{1}^{*}, \ldots$ there are an $M$-run $S_{0}, S_{1}, \ldots$ and sequences $i_{0}<i_{1}<\ldots, j_{0}<j_{1}<\ldots$ such that $i_{0}=j_{0}=0$ and $S_{i_{k}} \equiv S_{j_{k}}^{*}$ for each $k$ and either

- both runs terminate and their final states are the last pair of equivalent states, or

- both runs and both sequences $i_{0}<i_{1}<\ldots, j_{0}<j_{1}<\ldots$ are infinite.

The $M^{*}$-run $S_{0}^{*}, S_{1}^{*}, \ldots$ is said to simulate the $M$-run $S_{0}, S_{1}, \ldots$ The states $S_{i_{k}}, S_{j_{k}}^{*}$ are the corresponding states of interest. They represent the end points of the corresponding computation segments (those of interest) in Fig. 11 for which the equivalence is defined in terms of a relation between their corresponding locations (those of interest). The scheme shows that an ASM refinement allows one to combine in a natural way a change of the signature (through the definition of states and of their correspondence, of corresponding locations and of the equivalence of data) with a change of the control (defining the "flow of operations" appearing in the corresponding computation segments), thus integrating declarative and operational techniques and classical modularization concepts. 
The survey in [10] refers to numerous successful practical applications of the above definition, which generalizes other more restricted refinements notions in the literature [55156] and scales to the controlled and well documented development of large systems. In particular it supports modularizing ASM refinement correctness proofs aimed at mechanizable proof support, see [55]64]16]21].

\section{Summary of Work Done Using the ASM Method}

The ASM method to high-level system design and analysis, which is explained in the AsmBook 22, is characterized by the three notions of ASM, ASM ground model and ASM refinement.

ASMs are naturally defined as extension of Finite State Machines 13: just replace the two fixed FSM locations in and out, used for reading input and writing output symbols, by any set of readable and/or writable, possibly parameterized, locations $\left(l,\left(p_{1}, \ldots, p_{n}\right)\right)$ that may assume values of whatever types. Such sets of updatable locations represent arbitrarily complex abstract memory or states, what logicians call Tarski structures. Otherwise stated, ASMs are FSMs with generalized instructions of form If Condition Then Updates, where the FSMinput-event $i n=a$ is extended to an arbitrary first-order expression Condition and the FSM-output-operation out $:=b$ to an arbitrary set Updates of assignments $l\left(t_{1}, \ldots, t_{n}\right):=t$. This definition supports the intuitive understanding of ASMs as pseudo-code operating on abstract data structures.

Using ASMs as precise mathematical form of ground models 11 that are linked to compilable programs by ASM refinements [12, allows one to address the two sides of the software correctness problem in one framework, namely whether the ground model (read: the specification) faithfully reflects the intentions of the requirements and whether the code satisfies the ground model. For this purpose, the ASM method has been linked to a multitude of analysis methods, in terms of both experimental validation of models and mathematical verification of their properties.

The validation (testing) of ASM models is supported by numerous tools to mechanically execute ASMs (ASM Workbench [25], AsmGofer [59, an Asm2C++ compiler 60, C-based XASM [4, .NET-executable AsmL engine 32, CoreASM Execution Engine [31[30]). The verification of model properties is possible due to the mathematical character of ASMs, which means precision at the desired level of rigour. As a consequence any justification technique can be used, from proof sketches over traditional or formalized mathematical proofs [63 51] to tool supported proof checking or interactive or automatic theorem proving, e.g. by model checkers 68|26139, KIV [57] or PVS 28138. Also assertion-based techniques can be applied to the state-based run-time ASM models, thus combining so-called declarative (static logical) and operational (run-time state-based) methods and avoiding the straitjacket of purely axiomatic descriptions. Various combinations of such verification and validation methods have been supported and used also for the correctness analysis of compilers 29/49] and hardware 6665561/42]. 
As a consequence, the ASM method supports practical program design and analysis by the following four activities:

- formulate relevant ground model properties ("assertions as specifications in advance of code" [46, p.66]) in traditional mathematical terms, still free from any further burden and restriction that typically derive from additional concerns about a formalization in a specific logic language underlying a proof calculus one may want to use for logical deduction purposes,

- experimentally validate ground model properties by mental or mechanical simulation, performing experiments with the ground model as systematic attempts a) to "falsify" the model in the Popperian sense 52 against the to-be-encoded piece of reality, and b) to "validate" characteristic sets of scenarios, where "testing gives adequate assurance of serviceability" [46, p.69],

- mathematically verify desired ground model properties (e.g. their consistency), using traditional mathematical or (semi-) automated techniques,

- link ground models in a mathematically verifiable way to compilable code via ASM refinements.

\section{A Research Challenge and Some Milestones Ahead}

The main goal we want to propose, to lift Hoare's challenge from program verification to a discipline of verifiable system development, is a long-term and general methodological goal. It is clearly independent of the ASM system design and analysis method, but from the preceding sections it should have become clear that the ASM framework is appropriate to uniformly support the work on the overall challenge. The challenge is to provide (read: define and implement) an integrated tool support for hierarchies of mechanically verifiable and validatable model refinement patterns, which link in a provably correct and modular way the application-content of systems, as defined by ground models, to to-be-verified compilable programs. This implies extensions and enhancements of the currently available software development and analysis tools, targeted at combining in one project the definition of abstract models and their stepwise refinements with their simulation and verifications of their properties.

This main goal implies various subgoals, some of which we are going to describe as possible milestones of the overall challenge. The first group is related to the refinement method, the second group to the construction of ground models.

A refinement generator milestone consists in defining - and where possible mechanically generating - practical and provably correct model refinement schemes, which turn model properties into software interface assertions comprising behavioral component aspects. Such refinement schemes are to be used where run-time features are crucial for a satisfactory semantically founded correctness notion for code.

A refinement verifier milestone is to enhance leading mechanical verification systems by means to prove the correctness of model refinement steps. Such a verifier may exploit the modularity character of the underlying refinement schemes. 
There are various subgoals of this milestone. An example consists in linking ASMs to Event-B [1/2] along the lines of [14, so that the B verification tool set can be exploited to verify properties of ASMs and in particular the correctness of ASM refinement steps.

Another subgoal example consists in supporting verifications of the stepwise definition of programs written in widely used programming languages and their implementation on virtual machines. Such verifications are needed to close the model verification chain by linking verified abstract models to the generation of executable code. A concrete example in the literature has the form of mappings of Java to the Java Virtual Machine respectively of C\# to the .NET Common Language Runtime, which have been provided in 64 respectively in 17/34 with the goal of modeling and analyzing within a uniform framework the source language, the virtual machine and a compilation scheme linking the first to the second. This subgoal comes with a series of near milestones, e.g. to verify by existing mechanical theorem proving systems the following theorems, proved in 64] using layered ASM models for interpreters of Java and the JVM: Type safety of Java (Thm.8.4.1); Correctness of a Java2JVM compilation scheme (Thm.14.1.1); JVM invariants for the soundness of Bytecode Type Assignments (Thm.16.4.1); Completeness of the scheme for certifying Java2JVM compilation (Thm.16.5.1,16.5.2); Soundness of the bytecode verifier (Thm.17.1.1). A way to achieve this may be to extend the computer-based Java-subset verification documented in 5150.

An interesting practical outcome one can expect of such an endeavor for theorem proving systems is a set of reusable modular proof schemes that reflect hierarchies of layered abstract models, adding to the theorem-prover-oriented analysis provided in [54 55556] for the ASM refinement notion [12]. Schellhorn's analysis came out of the KIV verification, reported in [57/58, of the mathematical proof for the correctness of a compilation scheme of Prolog programs to Warren Abstract Machine code provided in [21], starting from the ASM model for ISO Prolog developed in 66|7/8|20]. This leads to another near milestone, namely reusing the Java/JVM-related proof schemes to establish the corresponding verifications for C\# and the .NET CLR, based upon their ASM models developed and verified in [17/48/34/36/33/37/35.

For language compilation there is also a compiler verification milestone, where real-life target processors take the place virtual machines occupy in the preceding milestones. It consists in developing methods supporting the verification of verifying compilers themselves in a general manner, adaptable for different source languages and target processors. A particular effort in this direction has been pursued in the Verifix [41] project, where ASM ground models were used extensively to describe the semantics of the underlying language and machines.

A related milestone consists in building a framework to guarantee forms of offdevice pre-verification of compiled code. For example for proving at compile time that the generated bytecode will pass the verifier one could extend the certifying compilation scheme developed for Java in [64, Sect.16.5], where the instructions are annotated with type information that can be and is used in 64. Thm. 16.5.1 
pg.266 sqq.] for the proof that the generated code is typable. This milestone is part of a more general challenge, namely to provide a practical theory to support the verification and validation of concepts and tools for generative programming techniques. Here the classical compile-link-run model of the semantics of programs has to be extended by a multistage- and meta-programming model for code, which is generated from components or code patterns or fragments, possibly written in low-level languages, according to directives that are expressed through metadata.

A refinement validation milestone consists in linking the refinement of ground models to model execution tools to make the generation and systematic comparison of corresponding test runs of abstract and refined machines possible. In particular relating system and unit level test results should be supported.

A runtime verification milestone consists in instrumenting current model execution tools to monitor the truth of selected properties at runtime, enabling in particular the exploration of ground models to detect undesired or hidden effects or missing behavior.

A re-engineering milestone is to define methods to extract ground models from legacy code as basis for analysis (and re-implementation where possible). The middle-size industrial case study described in [19] illustrates the feasibility of this goal.

A system certification milestone is to integrate ground model validation and analysis into industrial system certification processes. This effort can build upon the use that has been made of ASM ground models to formulate industrial standards, e.g. for the forthcoming standard of the Business Process Execution Language for Web Services [67, for the ITU-T standard for SDL-2000 [40, for the de facto standard for Java and the Java Virtual Machine [64], the ECMA standard for C\# and the .NET CLR 17/62, the IEEE-VHDL93 standard [18. This effort is certainly a long-term endeavor, but it appears to us to be both feasible and necessary to formulate the technical content of software reliability for embedded systems.

\section{Concluding Remark}

One reviewer asks what the advantages of the ASM method are over other approaches, whether it is "just a difference of notation" or whether there are "fundamental advantages". The conceptual simplicity of ASMs as FSMs updating arbitrary locations (read: general states), coupled to the use of standard algorithmic notation, constitutes a practical advantage: it makes ASMs understandable for application-domain experts and familiar to every software practitioner, thus supporting the mediation role ground models play for linking in an objectively checkable way informal requirements (read: natural-language descriptions of real-world phenomena) to mathematical models preceding compilable code. A further practical advantage of the ASM method is that it allows designers, programmers, verifiers and testers a) to exploit the abstraction/refinement pair, within one coherent mathematical framework, for a systematic separation of 
different concerns and b) to use any fruitful combination of whatever precise techniques are available - whether or not formalized within a specific logic or programming language or tool-to define, experimentally validate and mathematically verify a series of accurate system models leading to compilable code.

\section{References}

1. Abrial, J.-R.: Event based sequential program development: application to constructing a pointer program. In: Araki, K., Gnesi, S., Mandrioli, D. (eds.) FME 2003. LNCS, vol. 2805, pp. 51-74. Springer, Heidelberg (2003)

2. Abrial, J.-R.: Event driven distributed program construction. Version 6 (August 2004)

3. Abrial, J.-R.: On constructing large computerized systems (a position paper). In: Proc. VSTTE, ETH Zürich (October 2005)

4. Anlauff, M., Kutter, P.: Xasm Open Source (2001), http://www.xasm.org/

5. Betarte, G., Gimenez, E., Loiseaux, C., Chetali, B.: Formavie: Formal modelling and verification of the java card 2.1.1 security architecture. In: Proc. eSmart (2002)

6. Börger, E.: A logical operational semantics for full Prolog. In: Börger, E., Kleine Büning, H., Richter, M.M. (eds.) CSL 1989. LNCS, vol. 440, pp. 36-64. Springer, Heidelberg (1990)

7. Börger, E.: A logical operational semantics of full Prolog. In: Rovan, B. (ed.) MFCS 1990. LNCS, vol. 452, pp. 1-14. Springer, Heidelberg (1990)

8. Börger, E.: A logical operational semantics for full Prolog. In: Moschovakis, Y.N. (ed.) Part III: Built-in predicates for files, terms, arithmetic and input-output, Berkeley Mathematical Sciences Research Institute Publications. Logic From Computer Science, vol. 21, pp. 17-50. Springer, Heidelberg (1992)

9. Börger, E.: Logic programming: The Evolving Algebra approach. In: Pehrson, B., Simon, I. (eds.) IFIP 13th World Computer Congress. Technology/Foundations, vol. I, pp. 391-395. Elsevier, Amsterdam (1994)

10. Börger, E.: The origins and the development of the ASM method for high-level system design and analysis. J. Universal Computer Science 8(1), 2-74 (2002)

11. Börger, E.: The ASM ground model method as a foundation of requirements engineering. In: Dershowitz, N. (ed.) Verification: Theory and Practice. LNCS, vol. 2772, pp. 145-160. Springer, Heidelberg (2004)

12. Börger, E.: The ASM refinement method. Formal Aspects of Computing 15, 237 $257(2003)$

13. Börger, E.: The ASM method for system design and analysis. In: Gramlich, B. (ed.) FroCos 2005. LNCS (LNAI), vol. 3717, Springer, Heidelberg (2005)

14. Börger, E.: From Finite State Machines to Virtual Machines (Illustrating design patterns and event-B models). In: Cohors-Fresenborg, E., Schwank, I. (eds.) Präzisionswerkzeug Logik-Gedenkschrift zu Ehren von Dieter Rödding, Forschungsinstitut für Mathematikdidaktik Osnabr(2006) ISBN 3-925386-56-4

15. Börger, E., Dässler, K.: Prolog: DIN papers for discussion. ISO/IEC JTCI SC22 WG17 Prolog Standardization Document 58, National Physical Laboratory, Middlesex, England, (1990)

16. Börger, E., Durdanović, I.: Correctness of compiling Occam to Transputer code. Computer Journal 39(1), 52-92 (1996)

17. Börger, E., Fruja, G., Gervasi, V., Stärk, R.: A high-level modular definition of the semantics of C\#. Theoretical Computer Science 336(2-3), 235-284 (2005) 
18. Börger, E., Glässer, U., Müller, W.: The semantics of behavioral VHDL 1993 descriptions. In: EURO-DAC 1994. European Design Automation Conference with EURO-VHDL 1994, pp. 500-505. IEEE Computer Society Press, Los Alamitos (1994)

19. Börger, E., Päppinghaus, P., Schmid, J.: Report on a practical application of ASMs in software design. In: Gurevich, Y., Kutter, P.W., Odersky, M., Thiele, L. (eds.) ASM 2000. LNCS, vol. 1912, pp. 361-366. Springer, Heidelberg (2000)

20. Börger, E., Rosenzweig, D.: A mathematical definition of full Prolog. Science of Computer Programming 24, 249-286 (1995)

21. Börger, E., Rosenzweig, D.: The WAM - definition and compiler correctness. In: Beierle, C., Plümer, L. (eds.) Logic Programming: Formal Methods and Practical Applications. Studies in Computer Science and Artificial Intelligence, vol. 11, ch. 2, pp. 20-90. North-Holland, Amsterdam (1995)

22. Börger, E., Stärk, R.F.: Abstract State Machines. In: A Method for High-Level System Design and Analysis, Springer, Heidelberg (2003)

23. Brooks, F.P.J.: No silver bullet. Computer 20(4), 10-19 (1987)

24. Carnap, R.: The methodological character of theoretical concepts. In: Feigl, H., Scriven, M. (eds.) Minnesota Studies in the Philosophy of Science, vol. 2, pp. 3376. University of Minnesota Press (1956)

25. Del Castillo, G.: The ASM Workbench. A Tool Environment for Computer-Aided Analysis and Validation of Abstract State Machine Models. PhD thesis, Universität Paderborn, Germany (2001)

26. Del Castillo, G., Winter, K.: Model checking support for the ASM high-level language. In: Schwartzbach, M.I., Graf, S. (eds.) ETAPS 2000 and TACAS 2000. LNCS, vol. 1785, pp. 331-346. Springer, Heidelberg (2000)

27. Dijkstra, E.W.: Notes on structured programming. In: Dahl, O.-J., Dijkstra, E.W., Hoare, C.A.R. (eds.) Structured Programming, pp. 1-82. Academic Press, London (1972)

28. Dold, A.: A formal representation of Abstract State Machines using PVS. Verifix Technical Report Ulm/6.2, Universität Ulm, Germany (July 1998)

29. Dold, A., Gaul, T., Vialard, V., Zimmermann, W.: ASM-based mechanized verification of compiler back-ends. In: Glässer, U., Schmitt, P. (eds.) Proc. 5th Int. Workshop on ASMs, Magdeburg University, pp. 50-67 (1998)

30. Farahbod, R., et al.: The CoreASM Project, http://www.coreasm.org

31. Farahbod, R., Gervasi, V., Glässer, U.: CoreASM: An Extensible ASM Execution Engine. Fundamenta Informaticae XXI (2006)

32. Foundations of Software Engineering Group, Microsoft Research. AsmL (2001), http://research.microsoft.com/foundations/AsmL/

33. Fruja, N.G.: The Correctness of the Definite Assignment Analysis in C\#. J. Object Technology 3(9), 29-52 (2004)

34. Fruja, N.G.: A Modular Design for the.NET CLR Architecture. In: Beauquier, A.S.D., Börger, E. (eds.) 12th International Workshop on Abstract State Machines, ASM 2005, Paris, France, pp. 175-199 (March 2005)

35. Fruja, N.G.: Type Safety of Generics for the.NET Common Language Runtime. In: Sestoft, P. (ed.) ESOP 2006 and ETAPS 2006. LNCS, vol. 3924, pp. 325-341. Springer, Heidelberg (2006)

36. Fruja, N.G., Börger, E.: Analysis of the.NET CLR Exception Handling. In: Skala, V., Nienaltowski, P. (eds.) 3rd International Conference on .NET Technologies, NET 2005, Pilsen, Czech Republic, pp. 65-75 (May-June 2005)

37. Fruja, N.G., Börger, E.: Modeling the.NET CLR Exception Handling Mechanism for a Mathematical Analysis. Journal of Object Technology 5(3), 5-34 (2006) 
38. Gargantini, A., Riccobene, E.: Encoding Abstract State Machines in PVS. In: Gurevich, Y., Kutter, P.W., Odersky, M., Thiele, L. (eds.) ASM 2000. LNCS, vol. 1912, pp. 303-322. Springer, Heidelberg (2000)

39. Gawanmeh, A., Tahar, S., Winter, K.: Interfacing ASMs with the MDG tool. In: Börger, E., Gargantini, A., Riccobene, E. (eds.) ASM 2003. LNCS, vol. 2589, pp. 278-292. Springer, Heidelberg (2003)

40. Glässer, U., Gotzhein, R., Prinz, A.: Formal semantics of sdl-2000: Status and perspectives. Computer Networks 42(3), 343-358 (2003)

41. Goerigk, W., Dold, A., Gaul, T., Goos, G., Heberle, A., von Henke, F.W., Hoffmann, U., Langmaack, H., Pfeifer, H., Ruess, H., Zimmermann, W.: Compiler correctness and implementation verification: The verifix approach. In: Fritzson, P. (ed.) Int. Conf. on Compiler Construction, Proc. Poster Session of CC 1996, Linköping, Sweden (1996); IDA Technical Report LiTH-IDA-R-96-12

42. Habibi, A.: Framework for System Level Verification: The SystemC Case. PhD thesis, Concordia University, Montreal (July 2005)

43. Haeberer, A.M., Maibaum, T.S.E.: Scientific rigour, an answer to a pragmatic question: a linguistic framework for software engineering. In: International Conference on Software Engineering, Toronto, vol. 23 (2001)

44. Haeberer, A.M., Maibaum, T.S.E., Cengarle, M.V.: Knowing what requirements specifications specify (typoscript 2001)

45. Heimdahl, M.P.E.: Let's not forget validation. In: Meyer, B., Woodcock, J.C.P. (eds.) Verified Software: Theories, Tools, and Experiments (VSTTE 2005). LNCS, vol. 4171. Springer, Heidelberg (2008) (this volume)

46. Hoare, C.A.R.: The verifying compiler: A grand challenge for computing research. J. ACM 50(1), 63-69 (2003)

47. Jones, C.B.: What can we do (technically) to get the right specification. In: Meyer, B., Woodcock, J.C.P. (eds.) Verified Software: Theories, Tools, and Experiments (VSTTE 2005). LNCS, vol. 4171, pp. 64-69. Springer, Heidelberg (2008) (this volume)

48. Jula, H.V., Fruja, N.G.: An Executable Specification of C\#. In: Beauquier, A.S.D., Börger, E. (eds.) 12th International Workshop on Abstract State Machines, ASM 2005, Paris, France, pp. 275-287 (March 2005); University Paris 12

49. Kalinov, A., Kossatchev, A., Petrenko, A., Posypkin, M., Shishkov, V.: Using ASM specifications for compiler testing. In: Börger, E., Gargantini, A., Riccobene, E. (eds.) ASM 2003. LNCS, vol. 2589, p. 415. Springer, Heidelberg (2003)

50. Klein, G., Nipkow, T.: A machine-checked model for a Java-like language, virtual machine and compiler. ACM Trans. Prog. Lang. Syst. (2006)

51. Nanchen, S., Stärk, R.F.: A security logic for Abstract State Machines. TR 423 CS Dept., ETH Zürich (2003)

52. Popper, K.: Logik der Forschung. Zur Erkenntnishtoeire der modernen Naturwissenschaft. Wien (1935)

53. Glass, R.L.: Facts and Fallacies of Software Engineering. Addison-Wesley, Reading (2003)

54. Schellhorn, G.: Verifikation abstrakter Zustandsmaschinen. PhD thesis, Universität Ulm, Germany (1999)

55. Schellhorn, G.: Verification of ASM refinements using generalized forward simulation. J. Universal Computer Science 7(11), 952-979 (2001)

56. Schellhorn, G.: ASM refinement and generalizations of forward simulation in data refinement: A comparison. Theoretical Computer Science 336(2-3), 403-436 (2005)

57. Schellhorn, G., Ahrendt, W.: Reasoning about Abstract State Machines: The WAM case study. J. Universal Computer Science 3(4), 377-413 (1997) 
58. Schellhorn, G., Ahrendt, W.: The WAM case study: Verifying compiler correctness for Prolog with KIV. In: Bibel, W., Schmitt, P. (eds.) Automated Deduction - A Basis for Applications, volume III: Applications, pp. 165-194. Kluwer Academic Publishers, Dordrecht (1998)

59. Schmid, J.: Executing ASM specifications with AsmGofer, http://www.tydo.de/AsmGofer

60. Schmid, J.: Compiling Abstract State Machines to C++. J. Universal Computer Science 7(11), 1069-1088 (2001)

61. Schmid, J.: Refinement and Implementation Techniques for Abstract State Machines. PhD thesis, University of Ulm, Germany (2002)

62. Stärk, R.F., Börger, E.: An ASM specification of C\# threads and the.NET memory model. In: Zimmermann, W., Thalheim, B. (eds.) ASM 2004. LNCS, vol. 3052, Springer, Heidelberg (2004)

63. Stärk, R.F., Nanchen, S.: A logic for Abstract State Machines. J. Universal Computer Science 7(11), 981-1006 (2001)

64. Stärk, R.F., Schmid, J., Börger, E.: Java and the Java Virtual Machine: Definition, Verification, Validation. Springer, Heidelberg (2001)

65. Teich, J., Kutter, P., Weper, R.: Description and simulation of microprocessor instruction sets using ASMs. In: Gurevich, Y., Kutter, P.W., Odersky, M., Thiele, L. (eds.) ASM 2000. LNCS, vol. 1912, pp. 266-286. Springer, Heidelberg (2000)

66. Teich, J., Weper, R., Fischer, D., Trinkert, S.: A joint architecture/compiler design environment for ASIPs. In: Proc. Int. Conf. on Compilers, Architectures and Synthesis for Embedded Systems (CASES 2000), November 2000, pp. 26-33. ACM Press, San Jose (2000)

67. Vajihollahi, M.: High level specification and validation of the business process execution language for web services. Master's thesis, School of Computing Science at Simon Fraser University (March 2004)

68. Winter, K.: Model checking for Abstract State Machines. J. Universal Computer Science 3(5), 689-701 (1997)

69. Wirth, N.: Program development by stepwise refinement. Commun. ACM 14(4) (1971) 